\title{
Local Environment Affects Activity of Enzymes on 3D Molecular Scaffold Supporting Information
}

Yan Xiong ${ }^{1}$, James Huang ${ }^{2}$, Shih-Ting Wang ${ }^{3}$, Sufi Zafar ${ }^{4 *}$, Oleg Gang ${ }^{1,2,3 * *}$

1. Department of Chemical Engineering, Columbia University, New York, NY 10027, USA

2. Department of Applied Physics and Applied Mathematics, Columbia University, New York, NY 10027, USA

3. Center for Functional Nanomaterials, Brookhaven National Laboratory, Upton, NY 11973, USA

4. IBM T.J. Watson Research Center, Yorktown Heights, New York 10589, USA

*Sufi Zafar (Email: szafar@us.ibm.com)

**Oleg Gang (Email: og2226@columbia.edu)

\section{Table of Content}

\section{Part 1. Materials and Methods}
a. Schematic of Bipolar Junction Transistor (BJT) Device
b. Functionalization of Glucose Oxidase (GOx)
c. Comparisons of Oxidation Reaction among Weak Buffers
d. Dependence of pH Change on Proton Generation Using the BJT Sensor
e. Blank Control of Glucose Addition Using the BJT Sensor
f. GOx Activity Measurements via Horseradish Peroxidase (HRP)/ABTS Assay
g. Modelling of pH-Distance Profile near DNA Origami

\section{Part 2. Electron Microscopy Measurement}

a. Sample Preparation and Transmission Electron Microscopy

\section{Part 3. DNA Sequences}
a. Octahedral Scaffold
b. DNA Strands for GOx-OS Attachment 


\section{Part 1. Materials and Methods}

\section{a. Schematic of Bipolar Junction Transistor (BJT) Device}

The bipolar junction transistor (BJT) pH-based sensor is composed of three basic components (Figure S1): (i) a BJT device as the transducer, (ii) a TiN sensing surface and (iii) a reference electrode; both the sensing surface and the reference electrode are immersed in the studied aqueous solution. The voltage of collector $\left(V_{C}\right)$ is set to zero and the voltage of emitter $\left(V_{E}\right)$ can also provide sensing information as discuss below.

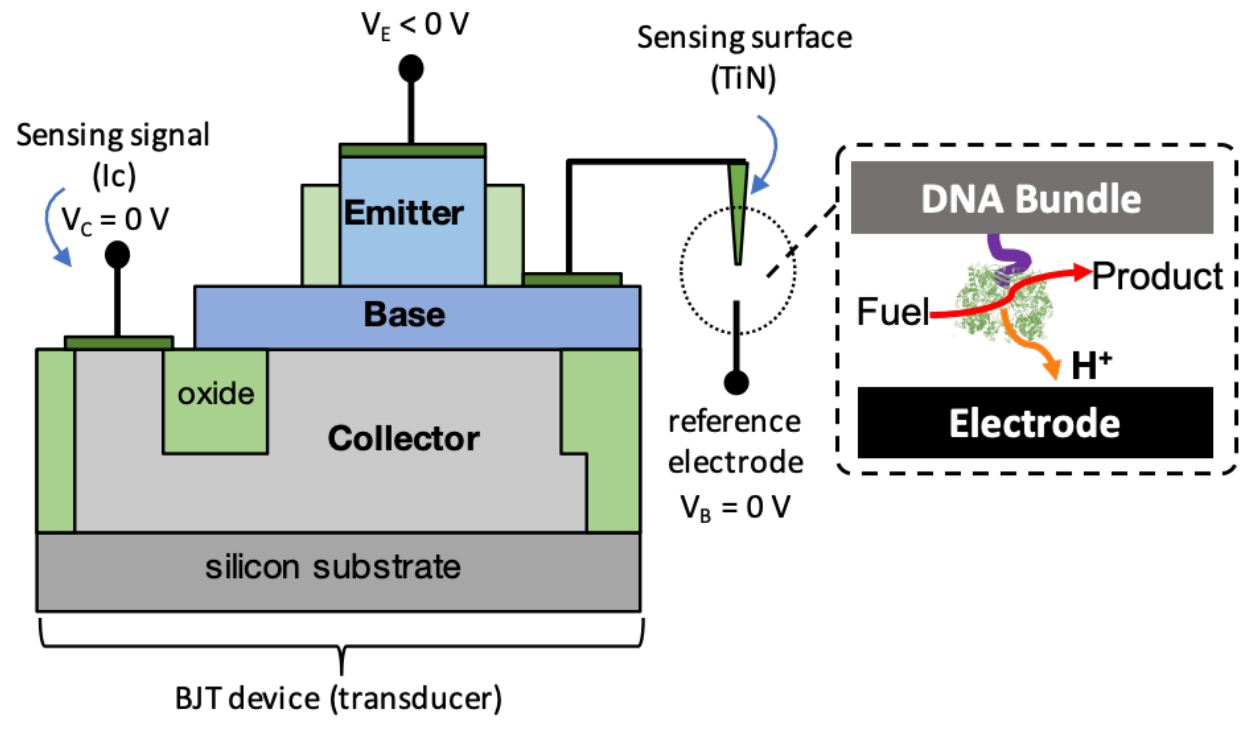

Figure S1. Schematic of the BJT device. 


\section{b. Functionalization of Glucose Oxidase}

Figures S2 and S3 show the characterization of DNA-attached glucose oxidase (GOx).

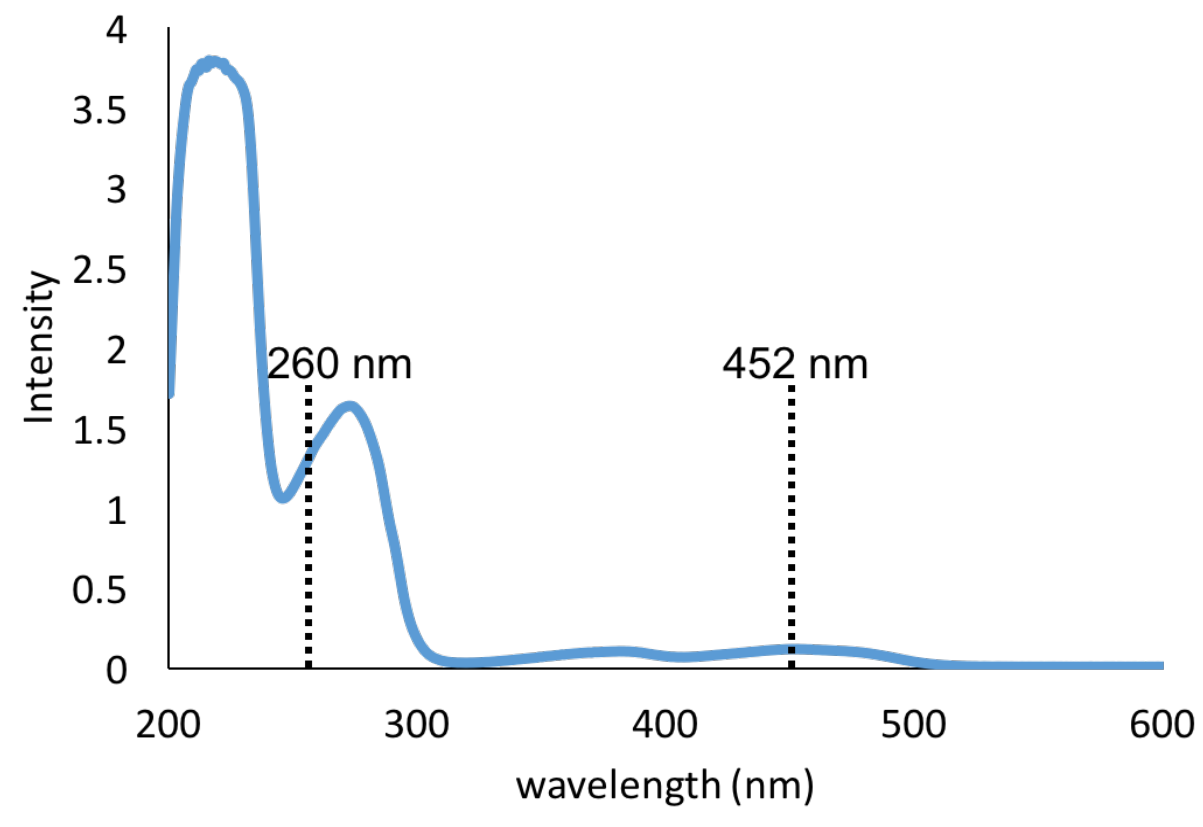

Figure S2. Ultraviolet-visible spectroscopy (UV-Vis) of DNA-GOx conjugates.

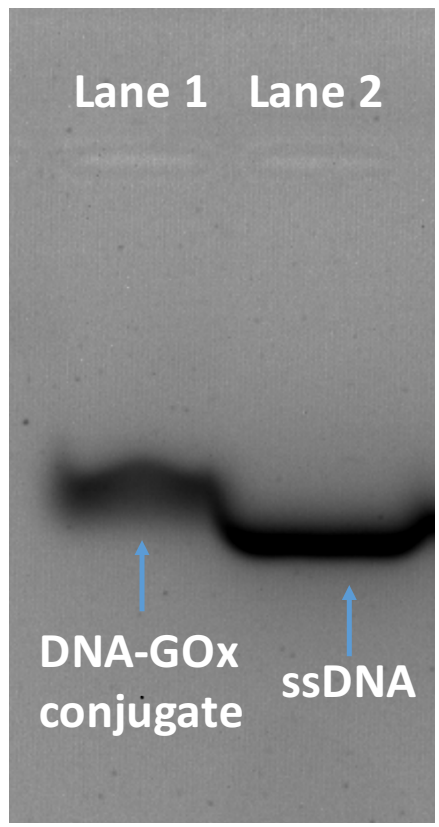

Figure S3. Agarose Gel image: Lane 1 is the sample of DNAGOx conjugates; Lane 2 is single stranded DNA (ssDNA). 
We compared the GOx activity before and after the DNA functionalization. The kinetics parameters (before functionalization: $K_{M}=7.2 \mathrm{mM}, k_{c a t}=128.1 \mathrm{~s}^{-1}$; after functionalization: $K_{M}=$ $10.2 \mathrm{mM}, k_{c a t}=114.3 \mathrm{~s}^{-1}$ ) were extracted from the experimental results (Fig. S4) based on Michaelis-Menten kinetics, indicating that the DNA functionalization did not significantly alter the intrinsic GOx activity.
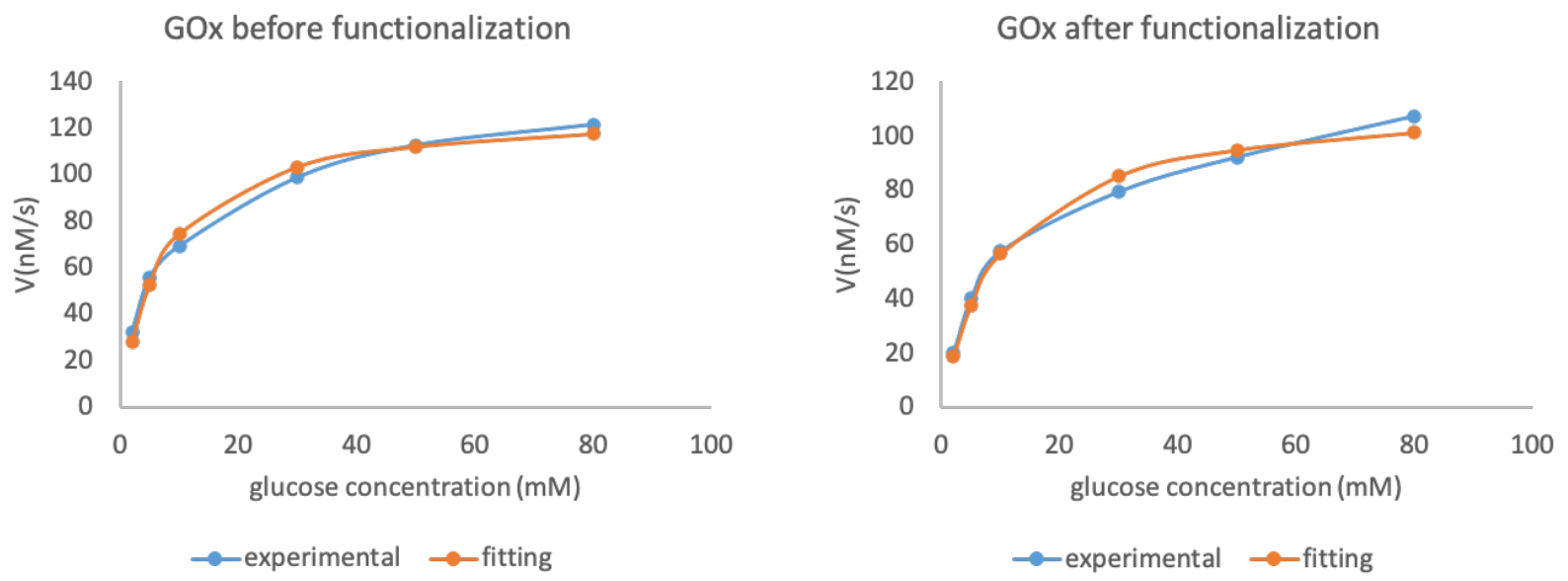

Figure S4. GOx activity before and after DNA functionalization measured by ABTS assay. Results were fitted based on Michaelis-Menten kinetics.

\section{c. Comparisons of Oxidation Reaction among Weak Buffers}

In order to study the buffering effect on the BJT probing, we prepared three buffers and then selected the most appropriate buffer for this study. The components of the three buffers are shown in the following Table S1:

Table S1. Components of customized weak Tris buffer

\begin{tabular}{lllll} 
Buffer Name & Tris & EDTA & $\mathbf{M g C l}_{2}$ & pH \\
\hline buffer 1 & $5 \mathrm{mM}$ & $0.5 \mathrm{mM}$ & $12.5 \mathrm{mM}$ & 7.45 \\
buffer 2 & $2 \mathrm{mM}$ & $0.2 \mathrm{mM}$ & $12.5 \mathrm{mM}$ & 7.35 \\
buffer 3 & $0.5 \mathrm{mM}$ & $0.05 \mathrm{mM}$ & $12.5 \mathrm{mM}$ & 7.35
\end{tabular}

As shown in Table S1, Tris and EDTA were used to stabilize the initial $\mathrm{pH}$ value of the working solution. Higher concentrations of these chemicals indicate a stronger buffering capacity. Therefore, the buffer capacity: buffer $1>2>3$. Note that the $12.5 \mathrm{mM} \mathrm{MgCl} 2$ worked to maintain 
the OS made of negatively charged DNA. The data presented in the paper was obtained using buffer 2 which was found to be the most appropriate buffer, as we discuss below.

First, we investigated the time dependence of proton accumulation $\left(\Delta\left[H^{+}(t)\right]=\left[H^{+}(t)\right]-\right.$ $\left.\left[H_{0}^{+}\right]\right)$and proton generation rate $\left(\frac{d\left[H^{+}(\mathrm{t})\right]}{d t}\right)$ in buffer 1 (Figures S5a-f) and buffer 2 (Figures 4a-f). Here, we show six measurements labeled as groups 1-6 where each group was assembled from different batches of the OS. As shown in Figures 3a-f, comparison between groups 1-3 (buffer 2, Figures 3a-f) and groups 4-6 (buffer 1, Figures S5a-f) indicates that buffer 2 with a weaker buffering capacity is more effective in amplifying the difference in proton boosting between GOxOS(A) and GOx-OS(B) than buffer 1. We propose that such difference could be caused by the buffering effect, where the protons, produced from the dissociation of buffer components and gluconic acid (GA), were partly absorbed by the buffer in order to restore equilibrium and maintain the solution $\mathrm{pH}$. Thus, a weaker buffering capacity (i.e., buffer 2) for neutralizing produced protons is able to intensify proton accumulation compared to concentrated buffer.

In order to further probe the buffering effect on proton sensing, we also measured the enzymatic activity of GOx-OS(A) in a more diluted buffer, buffer 3. As shown in Figure S4, pH jumps in the absence and presence of glucose were in the order of buffer $3>2>1$ similar to the trend of buffering strength. To ask the question of whether the buffer 3 could create a stable environment for enzymatic activity, we performed a conventional optical measurement using the 2,2'-azinobis(3-ethylbenzothiazoline-6-sulfonic acid) (ABTS) assay. Figure S5 shows that the catalytic activity of GOx-OS(A) in buffer 3 was only $60 \%$ of which in buffer 1 or 2 . The reduced GOx activity in buffer 3 implied that buffer 3 was too weak to maintain a proper $\mathrm{pH}$ for the enzymatic reaction. Note that our BJT sensor measurement also suggested a similar reduced $\frac{d\left[H^{+}(t)\right]}{d t}$ proportion of buffer 3 to buffer 1 (Figures S6b and S7).

It is noted that the absolute GA generation rate by the BJT sensor (Figure S6b) was much lower than hydrogen peroxide generation rate measured by the colorimetric method (Figure S7). This discrepancy could be explained by the interaction between GA and buffer system, implying that the BJT sensor, a label-free $\mathrm{pH}$ sensor, could not probe all the produced GA.

Overall, buffer 1 is too strong to distinguish the subtle difference between position A and B sets, while buffer 3 is too weak to stabilize enzymatic activity. Thus, buffer 2 is the most appropriate buffer for the BJT measurement, and it was employed in most of our study. 

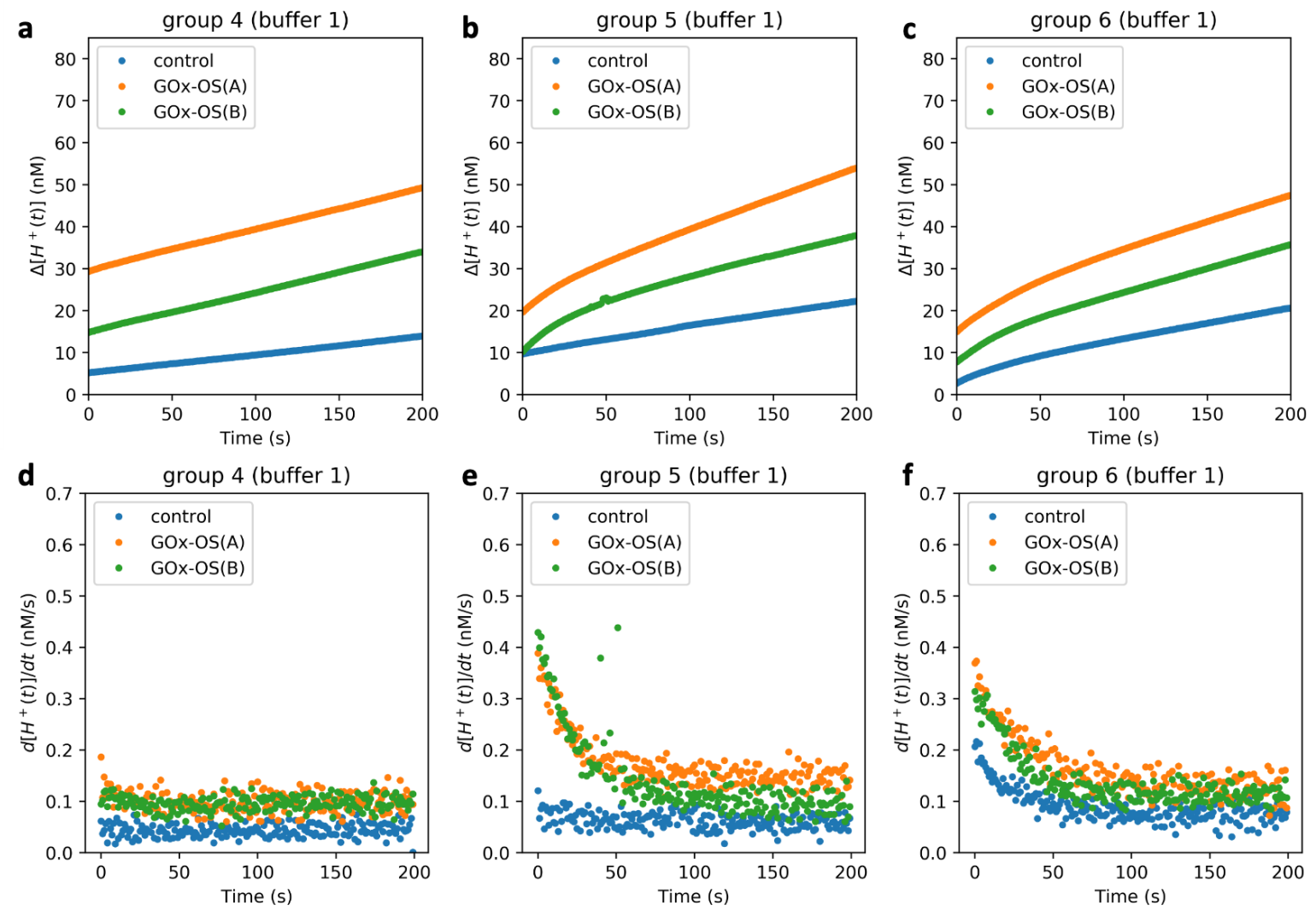

Figure S5. Real-time monitoring of proton accumulation $\left(\Delta\left[H^{+}(t)\right]=\left[H^{+}(t)\right]-\left[H_{0}^{+}\right]\right)$and proton generation rate $\left(\frac{d\left[H^{+}(t)\right]}{d t}\right)$ by the BJT sensor. Changes of $\Delta\left[H^{+}(t)\right](\mathrm{a}-\mathrm{c})$ and $\frac{d\left[\boldsymbol{H}^{+}(t)\right]}{d t}(\mathrm{~d}-\mathrm{f})$ within $200 \mathrm{~s}$ were monitored in diluted TE buffer 1 upon glucose addition, where three independent experiments (groups 4-6) were performed, respectively. Samples in different groups were made of OS from different batches. 

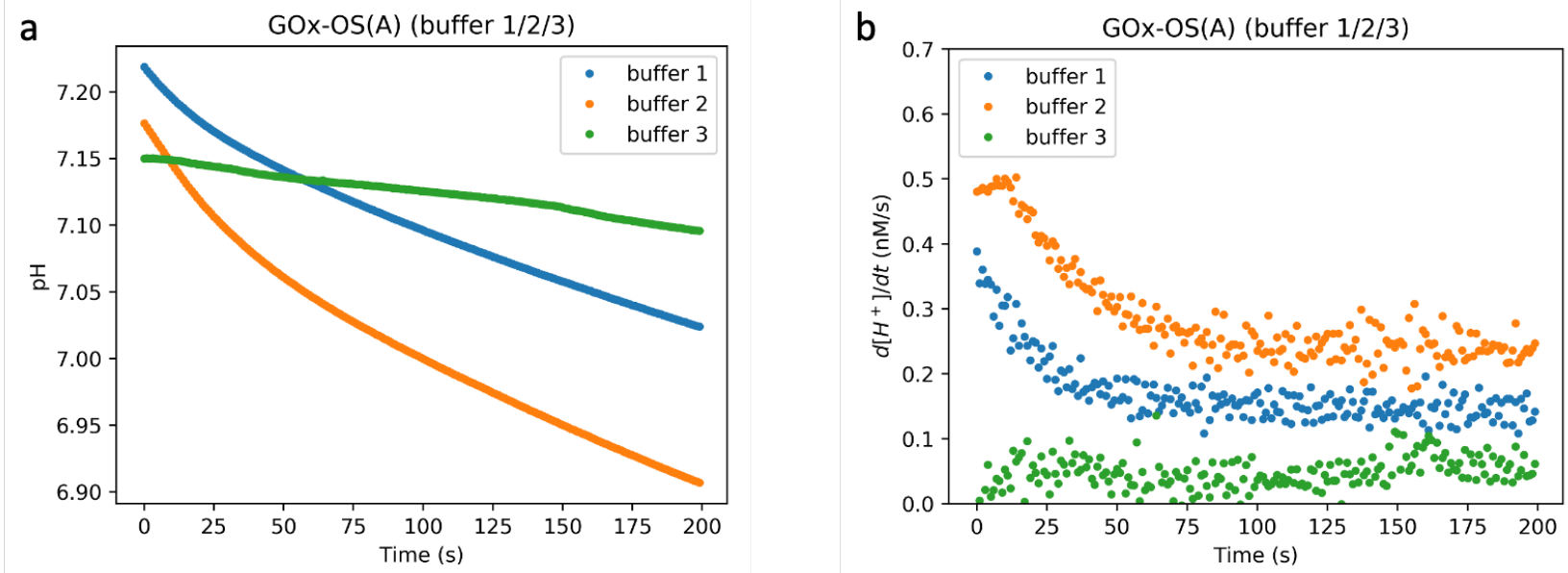

Figure S6. Comparison of GOx-OS(A) between buffers $1 / 2 / 3$ by the BJT sensor. (a) pH change of GOxOS(A) in buffers $1 / 2 / 3$ after glucose addition. Before glucose addition, the initial $\mathrm{pH}$ of buffers $1 / 2 / 3$ were 7.35, 7.35, and 7.45 respectively. Larger jump of $\mathrm{pH}$ value was observed in weaker buffer. (b) Proton generation rate of position A set in buffers $1 / 2 / 3$ buffer after glucose addition. In steady state, proton generation rate was in the order of buffer $2>1>3$.

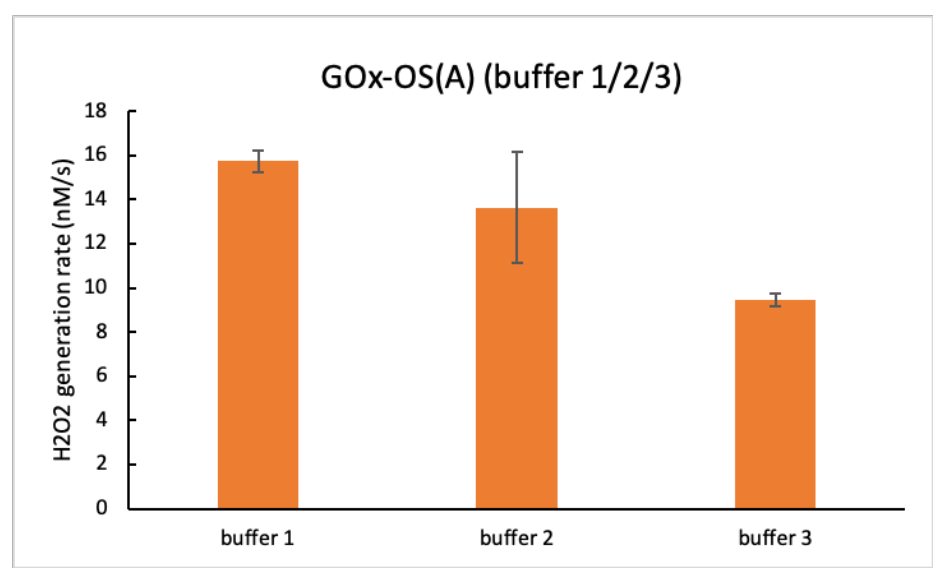

Figure S7. Comparison of ABTS assay of GOx-OS(A) between buffers $1 / 2 / 3$ by UV-Vis (see Table $\mathrm{S} 1)$. Hydrogen peroxide generation rates of three replicates were measured. GOx-OS(A) in buffer 3 shows only $60 \%$ of GOx-OS(A) in both buffers 1 and 2 . Samples in buffers 1 and 2 show similar catalytic activities. Error bars represent the s.d. of the three replicates.

\section{d. Dependence of pH Change on Proton Generation Using the BJT Sensor}

During the oxidation reaction, the $\mathrm{pH}$ of the buffer solution continued to drop because of proton generation, which in turn might affect the catalytic activity of GOx. In practice, the $\mathrm{pH}$ change (Figure S8) of two GOx-OS groups varied in a small range and it is evident that GOx-OS(A) 
preformed higher activity over GOx-OS(B) at the same $\mathrm{pH}$ value. Figure $\mathrm{S} 7$ also shows that the $\mathrm{pH}$ value of control group was about 0.2 less than GOx-OS groups, which may lead to about a maximum 10\% GOx activity variation (Figure 4c), ${ }^{1}$ while the BJT probing shows that GOx-OS groups showed about $200 \%$ activity enhancement than the control group. Thus, the $\mathrm{pH}$ change caused by the oxidation reaction was negligible compared to the effect of DNA scaffold.

Besides, we noticed that the bulk $\mathrm{pH}$ might alter the local $\mathrm{pH}$. Indeed, during the steady state (100-200 s measurement), around 0.1 unit of $\mathrm{pH}$ was changed, which did not significantly affect the local $\mathrm{pH}$ near DNA surface and thus did not affect our main conclusions.
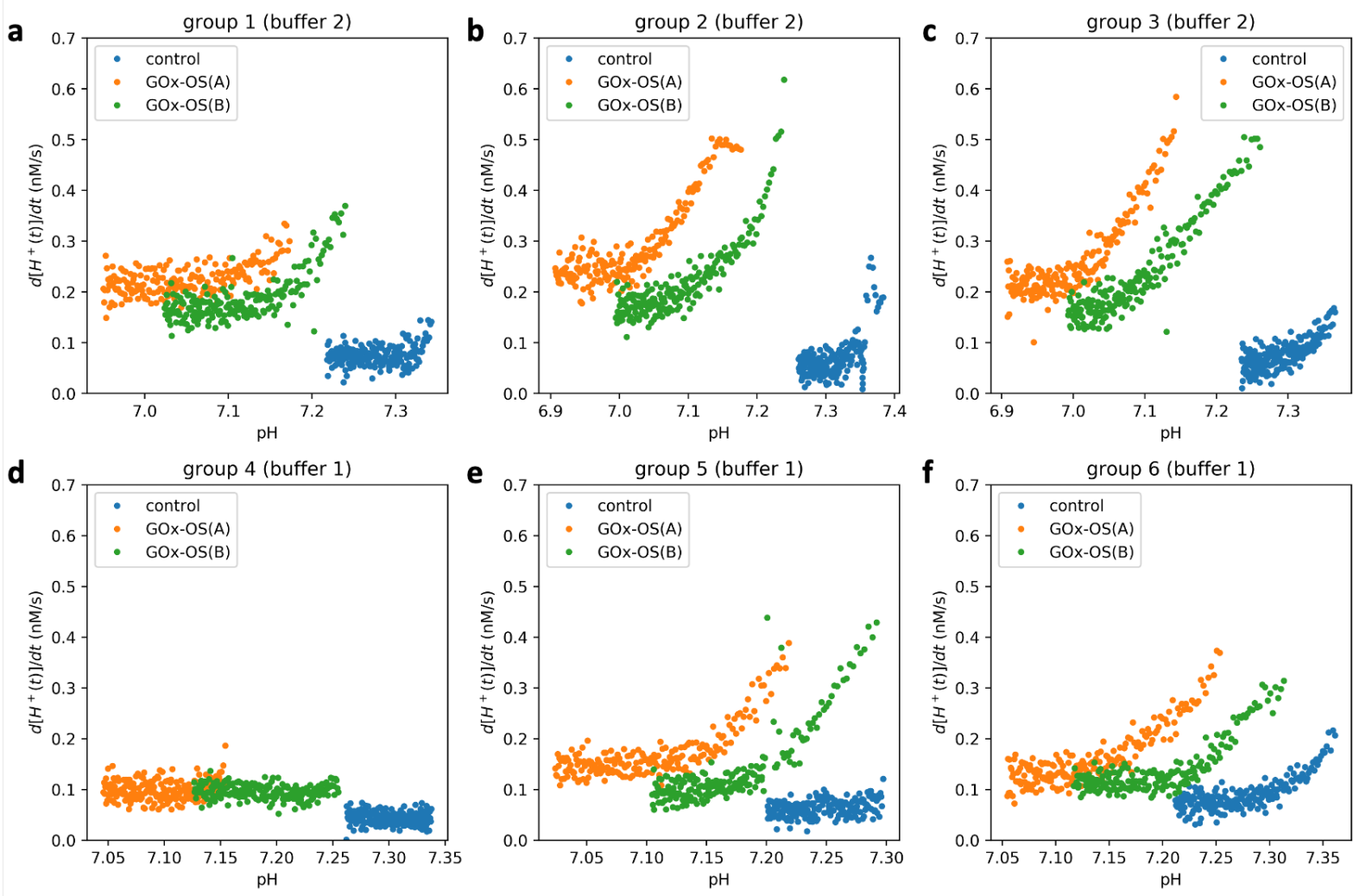

Figure S8. Plots of proton generation rates of OS-GOx versus $\mathrm{pH}$ change in buffers 1 and 2. (a), (b) and (c) show the relationship between proton generation rate and $\mathrm{pH}$ change of three replicates in buffer 2 during electronic measurements. (d), (e) and (f) show the relationship between proton generation rate and $\mathrm{pH}$ change of three replicates in buffer 1 during electronic measurements. Samples in different groups were made of origamis from different batches. 


\section{e. Blank Control of Glucose Addition Using the BJT Sensor}

In order to understand the disequilibrium of weak buffer caused by acidic additive, we added $32 \mu \mathrm{L}$ glucose solution (3 M glucose in diluted TE buffer) into $1168 \mu \mathrm{L}$ of the same buffer solution with neither DNA origami nor GOx. Figure S9 shows the $V_{E}$ curves before and after glucose addition.

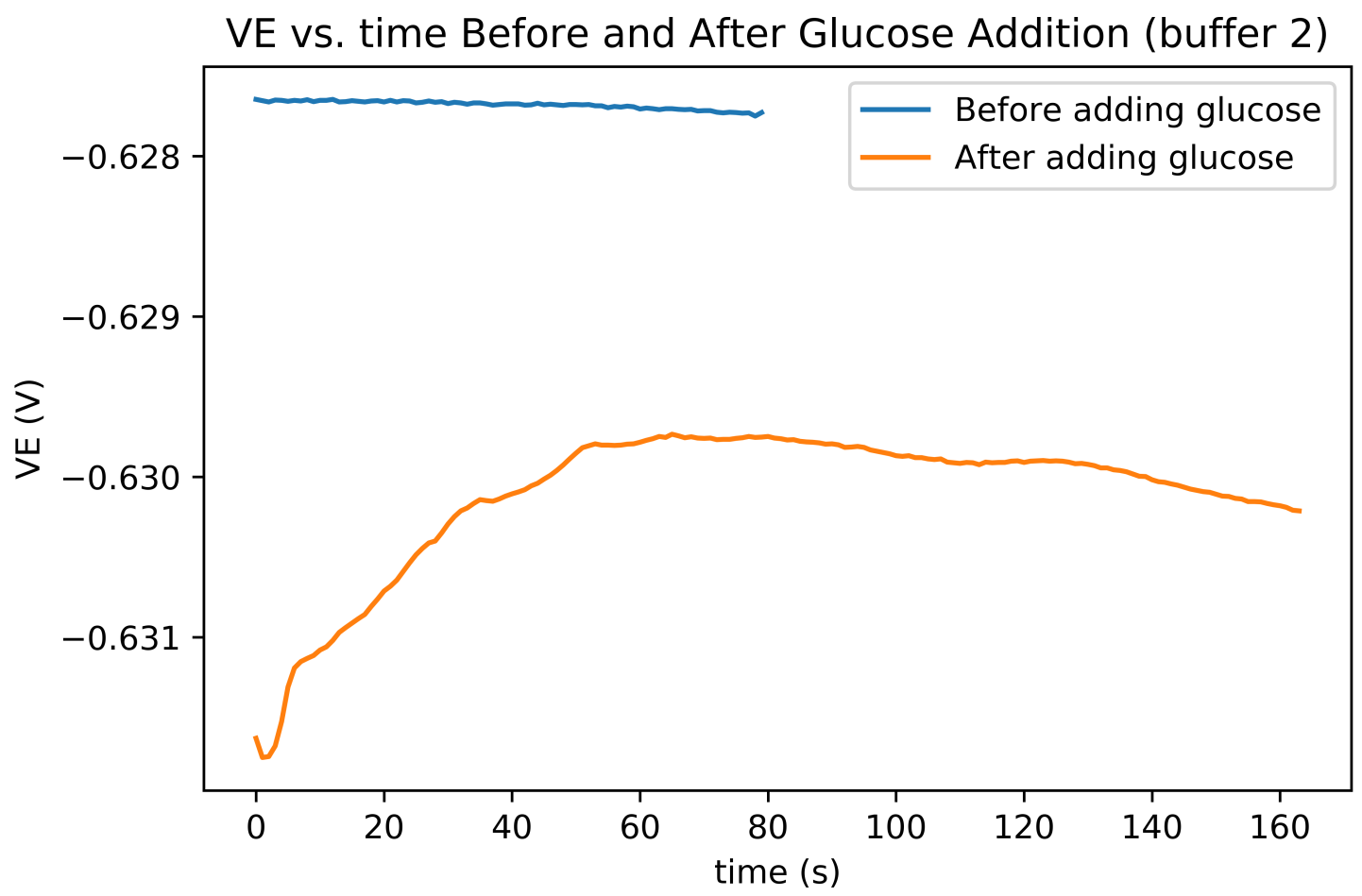

Figure S9. The dependence of $V_{E}$ on time before and after adding glucose. This blank control was in diluted TE buffer (buffer 2 in Table S1) and contained neither GOx nor DNA origami. Blue curve shows $V_{E}$ value before glucose addition where negligible fluctuation in OS-GOx solution was observed. Orange curve shows $V_{E}$ value after glucose addition where $V_{E}$ firstly jumped and then went up with a negative accumulation. After $60 \mathrm{~s}, V_{E}$ slightly dropped with a consistent rate. $V_{E}$ is the voltage applied at the emitter. The relationship between $I c$ and $V_{E}$ can be given as $I_{c}=$ $I_{0} \exp \left\{q\left(V_{B}+\psi_{s} I_{B} R-V_{E}\right) / k T\right\} . \psi_{s}$ is the sensing surface potential, $I_{0}$ is a constant dependent on device design parameters, $V_{B}$ is the base voltage applied at the reference electrode, $I_{B}$ is the base current flowing through the solution, $R$ is the resistance of the solution, $\left(I_{B} R\right)$ is the voltage drop in the solution, $k$ is the Boltzmann constant, $T$ is the device temperature in Kelvin, and $q$ is the electronic charge. 


\section{f. GOx Activity Measurements via Horseradish Peroxidase (HRP)/ABTS Assay}

Figure S10 shows the dependence of reaction rate on glucose concentration in buffers 1 and 2. Besides OS-GOx, unbound GOx and ssDNA-GOx have been put into test. This test is the control trial.

a

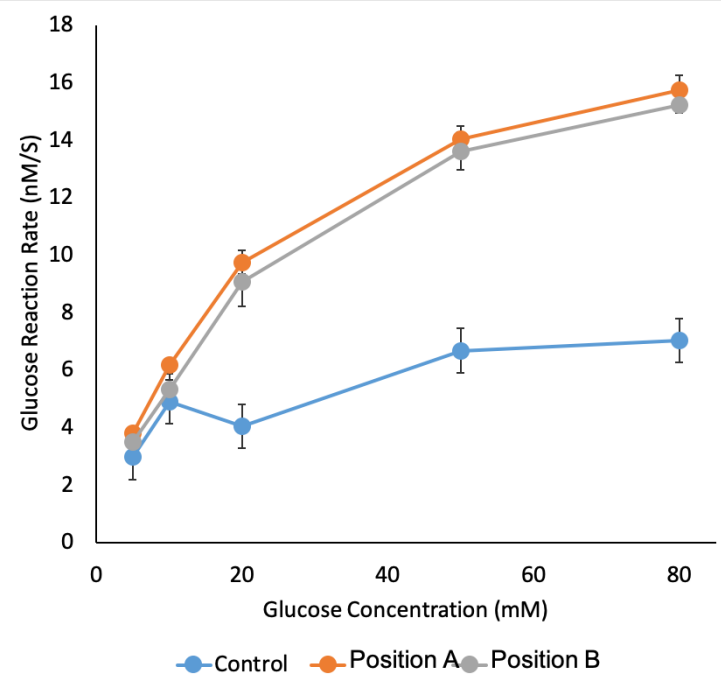

b

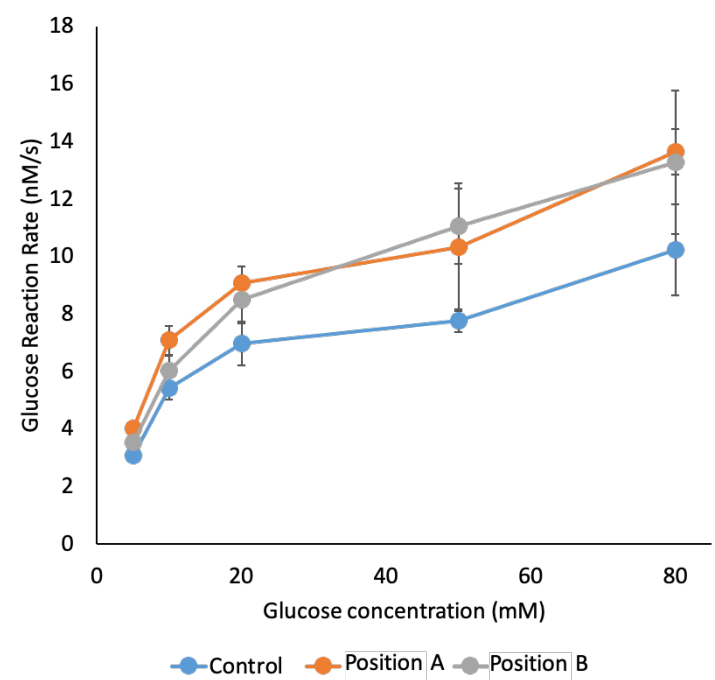

Figure S10. The dependence of glucose consumption rate on glucose concentration in (a) buffer 1 and (b) buffer 2 measured by HRP/ABTS assay. GOx concentration: $0.4 \mathrm{nM}$.

Figure 4c was plotted based on Table S2 collected by the HRP/ABTS assay (2 nM ssDNAGOx, $80 \mathrm{mM}$ glucose, $2 \mathrm{mM}$ ABTS). Note that there was no DNA origami in the solution.

Table S2. GOx activity dependence on pH using optical method

\begin{tabular}{|c|c|c|}
\hline $\mathrm{pH}$ & Normalized Reaction Rate & s.d. \\
\hline 8.26 & 0.326 & 0.052 \\
\hline 7.92 & 0.714 & 0.019 \\
\hline 7.38 & 1 & 0.016 \\
\hline 7.03 & 1.189 & 0.019 \\
\hline 6.50 & 1.264 & 0.088 \\
\hline 5.90 & 1.278 & 0.161 \\
\hline 5.42 & 1.389 & 0.088 \\
\hline
\end{tabular}




\section{g. Modelling of pH-Distance Profile near DNA Origami}

The pH-distance profile near charged DNA nanostructure was modeled using PoissonBoltzmann equation ${ }^{2}$ :

$$
\nabla^{2} \psi=-\frac{\rho_{e}}{\varepsilon_{r} \varepsilon_{0}}
$$

Where $\psi$ is local electric potential, $\rho_{e}$ is charge density, $\varepsilon_{r}$ is vacuum permittivity, $\varepsilon_{0}$ is dielectric constant of buffering solution.

The charge density can be calculated by $\rho_{e}=\sum_{i} z_{i} c_{i}$ using Boltzmann distribution:

$$
c_{i}=c_{i \infty} \exp \left(\frac{-z_{i} e \psi}{k_{B} T}\right)
$$

Where $c_{i}$ is local ion concentration of species $i, c_{i \infty}$ is bulk ion concentration of species $i, z_{i}$ is the charge on the species $i, e$ is the charge of a proton, $k_{B}$ is Boltzmann constant, $T$ is temperature.

Based on the two equations above, we established two models as the following:

(i) Assuming that the DNA origami is an infinite plate and symmetrical electrolytes in the solution, we reduced the Poisson-Boltzmann equation to Gouy-Chapman equation with boundary conditions:

$$
\begin{gathered}
\frac{d^{2} \psi}{d z^{2}}=-\frac{2 F c_{i \infty}}{\varepsilon_{r} \varepsilon_{0}} \sinh \left(-\frac{F \psi}{R T}\right) \\
\psi(z=0)=\psi_{0} \\
\psi(z=\infty)=0
\end{gathered}
$$

Where $\mathrm{z}$ is the distance from the DNA plate, $F$ is the Faraday constant.

The boundary conditions $\psi_{0}$ were estimated from the Grahame's Equation ${ }^{3}$ :

$$
\rho_{0}=\sqrt{8 c_{i \infty} \varepsilon R T} \sinh \left(\frac{F \psi_{0}}{2 R T}\right)
$$

$\rho_{0}$ is the surface charge density of DNA origami wall and it can be calculated by assuming that in DNA structure one phosphate group provides one elementary charge. Therefore, the surface charge density of ideal densest DNA plate is $\sim 2.0 \mathrm{q}_{e} / \mathrm{nm}^{2}$. Then, we roughly estimated that our DNA-bundle-like structure could have $\sim 0.2 q_{e} / \mathrm{nm}^{2}$ for the position A and $\sim 0.1 \mathrm{q}_{e} / \mathrm{nm}^{2}$ for the position B. 
The Boltzmann distribution also provided the relationship between $\mathrm{pH}$ profile and electric potential:

$$
p H(z)=p H_{0}+\frac{F \psi(z)}{2.3 R T}
$$

Where $R$ is the universal gas constant and $F$ is the Faraday constant.

Using the $c_{i \infty}$ in $2 \mathrm{mM}$ Tris buffer, we can plot the following $\mathrm{pH}$-distance profile:

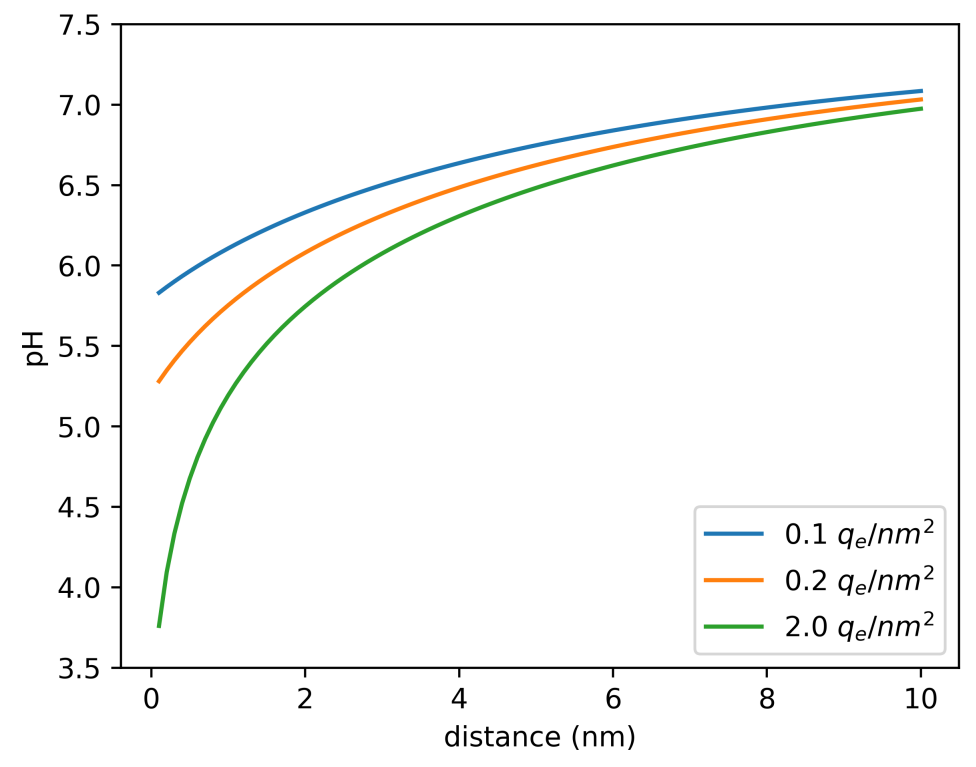

Figure S11. pH-distance profile near the DNA origami wall. Three curves with different surface charge densities were plotted where $2.0 q_{e} / \mathrm{nm}^{2}$ represents densest DNA plate, $0.2 q_{e} / \mathrm{nm}^{2}$ simulates position A, $0.1 q_{e} / \mathrm{nm}^{2}$ simulates position B.

We further estimated the effect of ionic strength on $\mathrm{pH}$ profile. We simplified the three buffers (Table S1) used in our work as Tris buffers, namely, buffer $1=5 \mathrm{mM}$ Tris, buffer $2=2 \mathrm{mM}$ Tris, buffer $3=0.5 \mathrm{mM}$ Tris. The following plot is obtained using $0.2 q_{e} / \mathrm{nm}^{2}$ surface charge density: 


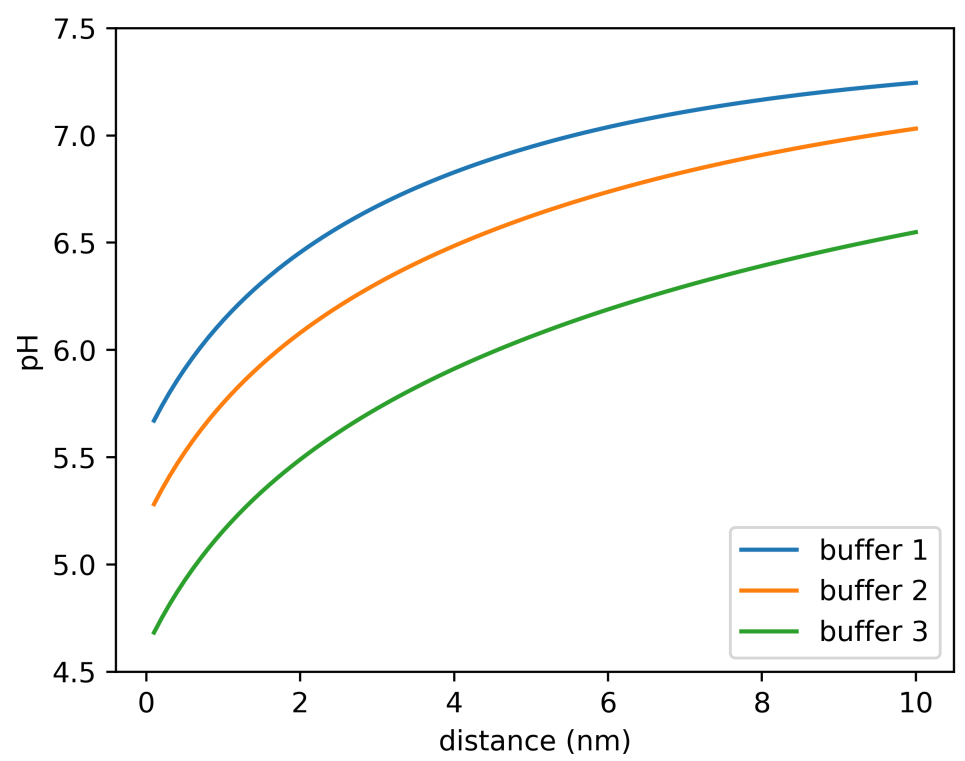

Figure S12. pH-distance profile near the DNA origami wall. Three curves with different buffers were plotted where buffer 1 is $5 \mathrm{mM}$ Tris, buffer 2 is $2 \mathrm{mM}$ Tris, buffer 3 is $0.5 \mathrm{mM}$ Tris.

From the prediction of two simulated $\mathrm{pH}$ profiles, higher charged surface and weaker buffer lead to lower local $\mathrm{pH}$. At a certain distance $(>8 \mathrm{~nm})$ from the DNA origami wall, the effect of charged surface is ambiguous, implying that the local ionic effect matters in a confined environment. Besides, it is noteworthy that even if weaker buffer displays stronger local pH effect, sufficient buffer capacity is required for maintaining stable $\mathrm{pH}$ for enzymatic reaction (Fig. S6).

(ii) Assuming the potential is small, we used the Debye-Hückel approximation to obtain simplyfied Poisson-Boltzmann equation:

$$
\nabla^{2} \psi=\kappa^{2} \psi
$$

Where $\lambda_{D}=1 / \kappa$ is the Debye length. In our system, the Debye length is estimated as $2 \mathrm{~nm}$.

Next, we picked three bundles and used partial differential equation toolbox in MATLAB to simulate the $\mathrm{pH}$ map around the three selected bundles (Fig. $4 \mathrm{~b}$ ). 


\section{Part 2. Electron Microscopy Measurement}

\section{a. Sample Preparation and Transmission Electron Microscopy}

Copper meshed grids were treated by plasma for $1 \mathrm{~min}$, followed by depositing wet samples on the discharged grid for $2.5 \mathrm{~min}$. Then the excess liquid was removed by a piece of filter paper. Next, $4 \mu \mathrm{L}$ uranyl acetate was added on the mesh grid for negative staining. The samples were dried in air prior to transmission electron microscopy (TEM).

In order to confirm the designed position for enzyme placement on the DNA scaffold, we used $5 \mathrm{~nm}$ DNA-grafted gold nanoparticle (AuNP) to hybridize with the sticky ends on OS at a ratio of 1 AuNP to 1.2 OS. Here, the DNA strands on the AuNPs had the same sequence as that on the GOx surface. Since there were multiple DNA strands coated on AuNP surface, in the position A set, one AuNP could cross-link with several OS (Figure S13a). As for ssDNA-GOx conjugate, according to UV-vis spectra, one ssDNA was attached to one protein, on average. Thus, the efficiency of single protein externally attached to single origami was higher than AuNP control set.

a
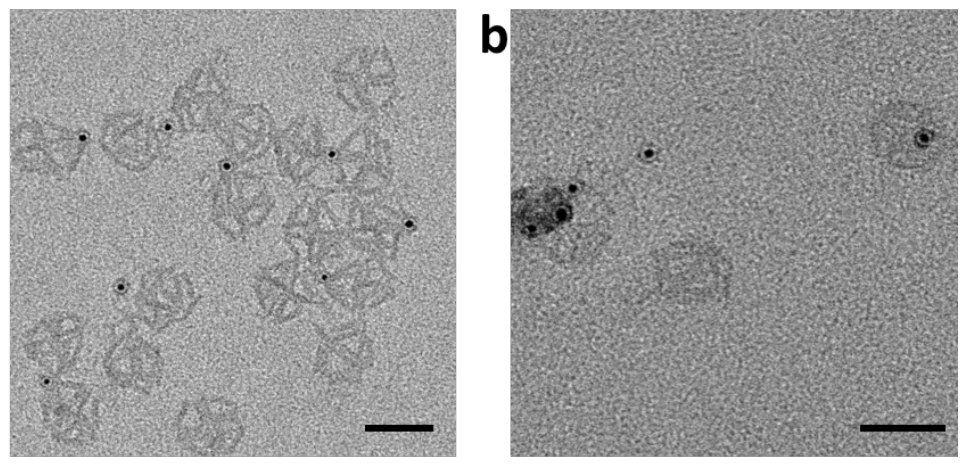

C
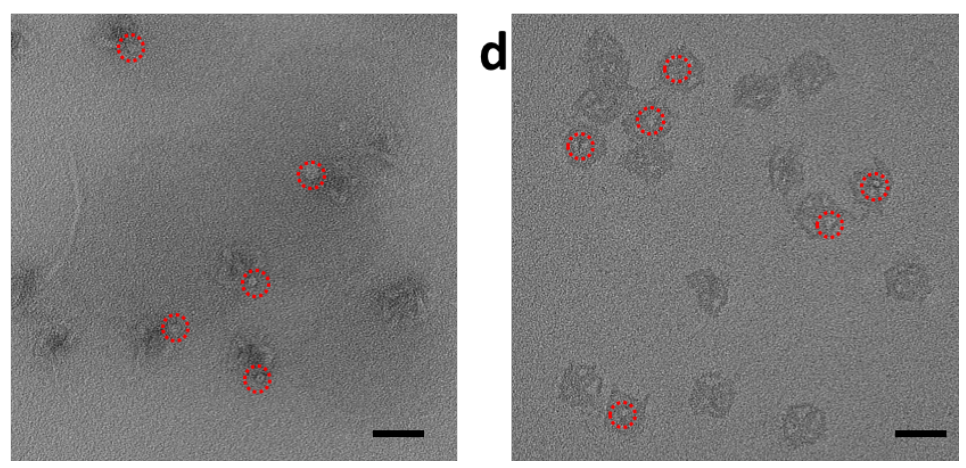

Figure S13. TEM images of OS-AuNP and GOx-OS conjugates. (a) AuNPs were bound to OS externally at position A; (b) AuNPs were bound to OS internally at position B; (c) GOx on OS at position A; (d) GOx on OS at position B. GOx was marked by red dash circles (scale bars: $50 \mathrm{~nm}$ ). 


\section{Part 3. DNA Sequences}

\section{a. Octahedral Scaffold}

Regular octahedral DNA staple strands:

R1

$\mathrm{R} 2$

R3

R4

R5

R6

R7

$\mathrm{R} 8$

R9

R10

R11

R12

R13

R14

R15

R16

R17

R18

R19

R20

$\mathrm{R} 21$

$\mathrm{R} 22$

R23

R24

$\mathrm{R} 25$

R26

R27

R28

R29

R30

R31

R32

R33

R34

R35

R36

R37

R38

R39

R40

R41
TCAAAGCGAACCAGACCGTTTTATATAGTC GCTTTGAGGACTAAAGAGCAACGGGGAGTT GTAAATCGTCGCTATTGAATAACTCAAGAA AAGCCTTAAATCAAGACTTGCGGAGCAAAT ATTTTAAGAACTGGCTTGAATTATCAGTGA GTTAAAATTCGCATTATAAACGTAAACTAG AGCACCATTACCATTACAGCAAATGACGGA ATTGCGTAGATTTTCAAAACAGATTGTTTG TAACCTGTTTAGCTATTTTCGCATTCATTC GTCAGAGGGTAATTGAGAACACCAAAATAG CTCCAGCCAGCTTTCCCCTCAGGACGTTGG GTCCACTATTAAAGAACCAGTTTTGGTTCC TAAAGGTGGCAACATAGTAGAAAATAATAA GATAAGTCCTGAACAACTGTTTAAAGAGAA GGTAATAGTAAAATGTAAGTTTTACACTAT TCAGAACCGCCACCCTCTCAGAGTATTAGC AAGGGAACCGAACTGAGCAGACGGTATCAT GTAAAGATTCAAAAGGCCTGAGTTGACCCT AGGCGTTAAATAAGAAGACCGTGTCGCAAG CAGGTCGACTCTAGAGCAAGCTTCAAGGCG CAGAGCCACCACCCTCTCAGAACTCGAGAG TTCACGTTGAAAATCTTGCGAATGGGATTT AAGTTTTAACGGGGTCGGAGTGTAGAATGG TTGCGTATTGGGCGCCCGCGGGGTGCGCTC GTCACCAGAGCCATGGTGAATTATCACCAATCAGAAAAGCCT GGACAGAGTTACTTTGTCGAAATCCGCGTGTATCACCGTACG CAACATGATTTACGAGCATGGAATAAGTAAGACGACAATAAA AACCAGACGCTACGTTAATAAAACGAACATACCACATTCAGG TGACCTACTAGAAAAAGCCCCAGGCAAAGCAATTTCATCTTC TGCCGGAAGGGGACTCGTAACCGTGCATTATATTTTAGTTCT AGAACCCCAAATCACCATCTGCGGAATCGAATAAAAATTTTT GCTCCATTGTGTACCGTAACACTGAGTTAGTTAGCGTAACCT AGTACCGAATAGGAACCCAAACGGTGTAACCTCAGGAGGTTT CAGTTTGAATGTTTAGTATCATATGCGTAGAATCGCCATAGC AAGATTGTTTTTTAACCAAGAAACCATCGACCCAAAAACAGG TCAGAGCGCCACCACATAATCAAAATCAGAACGAGTAGTATG GATGGTTGGGAAGAAAAATCCACCAGAAATAATTGGGCTTGA CTCCTTAACGTAGAAACCAATCAATAATTCATCGAGAACAGA AGACACCTTACGCAGAACTGGCATGATTTTCTGTCCAGACAA GCCAGCTAGGCGATAGCTTAGATTAAGACCTTTTTAACCTGT CCGACTTATTAGGAACGCCATCAAAAATGAGTAACAACCCCA 


\begin{tabular}{|c|c|}
\hline R42 & GTCCAATAGCGAGAACCAGACGACGATATTCAACGCAAGGGA \\
\hline $\mathrm{R} 43$ & CCAAAATACAATATGATATTCAACCGTTAGGCTATCAGGTAA \\
\hline R44 & AACAGTACTTGAAAACATATGAGACGGGTCTTTTTTAATGGA \\
\hline $\mathrm{R} 45$ & TTTCACCGCATTAAAGTCGGGAAACCTGATTTGAATTACCCA \\
\hline R46 & GAGAATAGAGCCTTACCGTCTATCAAATGGAGCGGAATTAGA \\
\hline R47 & ATAATTAAATTTAAAAAACTTTTTCAAACTTTTAACAACGCC \\
\hline $\mathrm{R} 48$ & GCACCCAGCGTTTTTTATCCGGTATTCTAGGCGAATTATTCA \\
\hline R49 & GGAAGCGCCCACAAACAGTTAATGCCCCGACTCCTCAAGATA \\
\hline $\mathrm{R} 50$ & GTTTGCCTATTCACAGGCAGGTCAGACGCCACCACACCACCC \\
\hline R51 & CGCGAGCTTAGTTTTTCCCAATTCTGCGCAAGTGTAAAGCCT \\
\hline $\mathrm{R} 52$ & AGAAGCAACCAAGCCAAAAGAATACACTAATGCCAAAACTCC \\
\hline R53 & ATTAAGTATAAAGCGGCAAGGCAAAGAAACTAATAGGGTACC \\
\hline R54 & CAGTGCCTACATGGGAATTTACCGTTCCACAAGTAAGCAGAT \\
\hline R55 & ATAAGGCGCCAAAAGTTGAGATTTAGGATAACGGACCAGTCA \\
\hline R56 & TGCTAAACAGATGAAGAAACCACCAGAATTTAAAAAAAGGCT \\
\hline R57 & CAGCCTTGGTTTTGTATTAAGAGGCTGACTGCCTATATCAGA \\
\hline R58 & CGGAATAATTCAACCCAGCGCCAAAGACTTATTTTAACGCAA \\
\hline R59 & CGCCTGAATTACCCTAATCTTGACAAGACAGACCATGAAAGA \\
\hline R60 & ACGCGAGGCTACAACAGTACCTTTTACAAATCGCGCAGAGAA \\
\hline R61 & CAGCGAACATTAAAAGAGAGTACCTTTACTGAATATAATGAA \\
\hline R62 & GGACGTTTAATTTCGACGAGAAACACCACCACTAATGCAGAT \\
\hline R63 & AAAGCGCCAAAGTTTATCTTACCGAAGCCCAATAATGAGTAA \\
\hline R64 & GAGCTCGTTGTAAACGCCAGGGTTTTCCAAAGCAATAAAGCC \\
\hline R65 & AATTATTGTTTTCATGCCTTTAGCGTCAGATAGCACGGAAAC \\
\hline R66 & AAGTTTCAGACAGCCGGGATCGTCACCCTTCTGTAGCTCAAC \\
\hline R67 & ACAAAGAAATTTAGGTAGGGCTTAATTGTATACAACGGAATC \\
\hline R68 & AACAAAAATAACTAGGTCTGAGAGACTACGCTGAGTTTCCCT \\
\hline R69 & CATAACCTAAATCAACAGTTCAGAAAACGTCATAAGGATAGC \\
\hline R70 & CACGACGAATTCGTGTGGCATCAATTCTTTAGCAAAATTACG \\
\hline R71 & CCTACCAACAGTAATTTTATCCTGAATCAAACAGCCATATGA \\
\hline $\mathrm{R} 72$ & GATTATAAAGAAACGCCAGTTACAAAATTTACCAACGTCAGA \\
\hline $\mathrm{R} 73$ & AGTAGATTGAAAAGAATCATGGTCATAGCCGGAAGCATAAGT \\
\hline R74 & TAGAATCCATAAATCATTTAACAATTTCTCCCGGCTTAGGTT \\
\hline R75 & AAAGGCCAAATATGTTAGAGCTTAATTGATTGCTCCATGAGG \\
\hline R76 & CCAAAAGGAAAGGACAACAGTTTCAGCGAATCATCATATTCC \\
\hline R77 & GAAATCGATAACCGGATACCGATAGTTGTATCAGCTCCAACG \\
\hline R78 & TGAATATTATCAAAATAATGGAAGGGTTAATATTTATCCCAA \\
\hline R79 & GAGGAAGCAGGATTCGGGTAAAATACGTAAAACACCCCCCAG \\
\hline R80 & GGTTGATTTTCCAGCAGACAGCCCTCATTCGTCACGGGATAG \\
\hline R81 & CAAGCCCCCACCCTTAGCCCGGAATAGGACGATCTAAAGTTT \\
\hline $\mathrm{R} 82$ & TGTAGATATTACGCGGCGATCGGTGCGGGCGCCATCTTCTGG \\
\hline $\mathrm{R} 83$ & CATCCTATTCAGCTAAAAGGTAAAGTAAAAAGCAAGCCGTTT \\
\hline R84 & CAGCTCATATAAGCGTACCCCGGTTGATGTGTCGGATTCTCC \\
\hline $\mathrm{R} 85$ & CATGTCACAAACGGCATTAAATGTGAGCAATTCGCGTTAAAT \\
\hline R86 & AGCGTCACGTATAAGAATTGAGTTAAGCCCTTTTTAAGAAAG \\
\hline R87 & TATAAAGCATCGTAACCAAGTACCGCACCGGCTGTAATATCC \\
\hline
\end{tabular}




$\begin{array}{rr}\text { R88 } & \text { ATAGCCCGCGAAAATAATTGTATCGGTTCGCCGACAATGAGT } \\ \text { R89 } & \text { AGACAGTTCATATAGGAGAAGCCTTTATAACATTGCCTGAGA } \\ \text { R90 } & \text { AACAGGTCCCGAATTGCATCAAAAAGATCTTTGATCATCAG } \\ \text { R91 } & \text { ACTGCCCTGCCCCGTGCAGCAAGCGGCAACAGCTTTTCT } \\ \text { R92 } & \text { TCAAAGGGAGATAGCCCTATAAATCAAGACAACAACCATCG } \\ \text { R93 } & \text { GTAATACGCAAACATGAGAGATCTACAACTAGCTGAGGCCGG } \\ \text { R94 } & \text { GAGATAACATTAGAAGAATAACATAAAAAGGAAGGATTAGGA } \\ \text { R95 } & \text { CAGATATTACCTGAATACCAAGTTACAATCGGGAGCTATTTT } \\ \text { R96 } & \text { CATATAACTAATGAACACAACATACGAGCTGTTTCTTGGGG } \\ \text { R97 } & \text { ATGTTTTGCTTTGATCGGAACGAGGGTACTTTTTCTTTTGATAAGAGGTCATT } \\ \text { R98 } & \text { GGGGTGCCAGTTGAGACCATTAGATACAATTTTCACTGTGTGAAATTGTTATCC } \\ \text { R99 } & \text { CTTCGCTGGGCGCAGACGACAGTATCGGGGCACCGTCGCCATTCAGGCTGCGCA } \\ \text { R100 } & \text { TCAGAGCTGGGTAAACGACGGCCAGTGCGATCCCCGTAGTAGCATTAACATCCA } \\ \text { R101 } & \text { TTAGCGGTACAGAGCGGGAGAATTAACTGCGCTAATTTCGGAACCTATTATTCT } \\ \text { R102 } & \text { GATATTCTAAATTGAGCCGGAACGAGGCCCAACTTGGCGCATAGGCTGGCTGAC } \\ \text { R103 } & \text { TGTCGTCATAAGTACAGAACCGCCACCCATTTCACAGTACAAACTACAACGCC } \\ \text { R104 } & \text { CGATTATAAGCGGAGACTTCAAATATCGCGGAAGCCTACGAAGGCACCAACCTA } \\ \text { R105 } & \text { AACATGTACGCGAGTGGTTTGAAATACCTAAACACATTCTTACCAGTATAAAGC } \\ \text { R106 } & \text { GTCTGGATTTTGCGTTTTAAATGCAATGGTGAGAAATAAATTAATGCCGGAGAG } \\ \text { R107 } & \text { GCCTTGAATCTTTTCCGGAACCGCCTCCCAGAGCCCAGAGCCGCCGCCAGCATT } \\ \text { R108 } & \text { CGCTGGTGCTTTCCTGAATCGGCCAACGAGGGTGGTGATTGCCCTTCACCGCCT } \\ \text { R109 } & \text { TGATTATCAACTTTACAACTAAAGGAATCCAAAAAGTTTGAGTAACATTATCAT } \\ \text { R110 } & \text { ACATAACTTGCCCTAACTTTAATCATTGCATTATAACAACATTATTACAGGTAG } \\ \text { R111 } & \text { GTAGCGCCATTAAATTGGGAATTAGAGCGCAAGGCGCACCGTAATCAGTAGCGA } \\ \text { R112 } & \text { TTATTTTACCGACAATGCAGAACGCGCGAAAAATCTTTCCTTATCATTCCAAG } \\ \text { R113 } & \text { TTTCAATAGAAGGCAGCGAACCTCCGATTAGTTGAAACAATAACGGATTCGCC } \\ \text { R114 } & \text { GGGCGACCCCAAAAGTATGTTAGCAAACTAAAAGAGTCACAATCAATAGAAAAT } \\ \text { R115 } & \text { AGCCGAAAGTCTCTCTTTTGATGATACAAGTGCCTTAAGAGCAAGAAACAATGA } \\ \text { R116 } & \text { GTGGGAAATCATATAAATATTTAAATTGAATTTTGTCTGGCCTTCCTGTAGCC } \\ \text { R117 } & \text { CCCACGCGCAAAATGGTTGAGTGTTGTTCGTGGACTTGCTTTCGAGGTGAATTT } \\ \text { R118 } & \text { ATGACCACTCGTTTGGCTTTTGCAAAAGTTAGACTATATTCATTGAATCCCCCT } \\ \text { R119 } & \text { TCCAAATCTTCTGAATTATTTGCACGTAGGTTTAACGCTAACGAGCGTCTTTCC } \\ \text { R120 } & \text { GGGTTATTTAATTACAATATATGTGAGTAATTAATAAGAGTCAATAGTGAATTT }\end{array}$

\section{b. DNA Strands for GOx-OS Attachment}

For external set, add one more staple into the 120 staples presented above:

TTTGCGGATGGCCAACTAAAGTACGGGCTTGCAGCTACAGAGTTTATCCATACTACC ATCTAC

For internal set, use the following staple to replace R54:

CAGTGCCTACATGGGAATTTACCGTTCCACAAGTAAGCAGATTTTATCCATACTACC ATCTAC

The DNA strands attached to GOx were: /5ThioMC6-D/TTTTTGTAGATGGTAGTATGGAT 


\section{References}

1. Zhang, Y.; Tsitkov, S.; Hess, H., Proximity does not Contribute to Activity Enhancement in the Glucose Oxidase-Horseradish Peroxidase Cascade. Nat. Commun. 2016, 7, 13982.

2. Sharp, K.; Honig, B., Calculating Total Electrostatic Energies with the Nonlinear PoissonBoltzmann Equation. J. Phys. Chem. 1990, 94, 7684-7692.

3. Hans-Jurgen, B.; Graf, K.; Kappl, M., Physics and Chemistry of Interfaces. Wiley-VCH: Germany, 2006. 